\title{
RESISTENCIA ANTIBIÓTICA DE Streptococcuspneumoniae EN PORTADORES NASOFARÍNGEOS SANOS DE SIETE REGIONES DEL PERÚ
}

Nancy Torres ${ }^{1, a}$, Ricardo Velásquez ${ }^{1, a}$, Erik H. Mercado ${ }^{2, b}$, Martha Egoavil ${ }^{1, a}$, Gertrudis Horna ${ }^{2, c}$, Lida Mejía ${ }^{2, c}$, María E. Castillo ${ }^{1,3, d}$, Eduardo Chaparro ${ }^{1,4, d}$, Roger Hernández ${ }^{1,4, d}$, Wilda Silva ${ }^{5, d}$, Francisco E. Campos ${ }^{6, d}$, Andrés Sáenz ${ }^{7, d}$, Félix Hidalgo ${ }^{8, d}$, Carolina Letona $a^{9, d}$, Ángel G. Valencia ${ }^{10, d}$, Rosario Cerpa ${ }^{11, d}$, Bernardo López-de-Romaña ${ }^{12, d}$, Berenice Torres ${ }^{1, a}$, Fiorella Castillo ${ }^{1, a}$, Andrea Calle ${ }^{1, a}$, Synthia Rabanal ${ }^{1, a}$, Jackeline Pando ${ }^{13, d}$, Elizabeth Lacroix ${ }^{14}$, Isabel Reyes ${ }^{15, d}$, Humberto Guerra ${ }^{2, e}$, Theresa J. Ochoa ${ }^{1,2,16, d}$

\section{RESUMEN}

Objetivos. Determinar el patrón de susceptibilidad antibiótica de cepas de Streptococcus pneumoniae aisladas de portadores nasofaríngeos sanos menores de 2 años de siete regiones del Perú. Materiales y métodos. Entre el 2007 y 2009 se tomaron muestras de hisopado nasofaríngeo a 2123 niños sanos entre 2 y 24 meses de edad en los consultorios de crecimiento y desarrollo (CRED) y vacunación de hospitales y centros de salud de Lima, Piura, Cusco, Abancay, Arequipa, Huancayo, e lquitos. Se determinó la resistencia a diez antibióticos mediante la prueba de disco-difusión de las cepas de neumococo aisladas. Resultados. Se aislaron 572 cepas. Se encontró altas tasas de resistencia a cotrimoxazol (58\%); penicilina (52,2\% no-sensibles); tetraciclina (29,1\%); azitromicina $(28,9 \%)$, y eritromicina $(26,3 \%)$. La resistencia a cloranfenicol fue baja $(8,8 \%)$. Se encontró $29,5 \%$ de multirresistencia. La resistencia a la azitromicina y a la penicilina fue diferente en las siete regiones $(p<0,05)$, hallándose el mayor porcentaje de cepas no-sensibles a penicilina en Arequipa $(63,6 \%)$, mientras que el menor fue en Cusco $(23,4 \%)$. Conclusiones. Los elevados niveles de resistencia encontrados para penicilina, cotrimoxazol y macrólidos en cepas de neumococo aisladas de portadores sanos en todas las regiones estudiadas, y su asociación con uso previo de antibióticos, representan un importante problema de salud pública en nuestro país. Esto resalta la necesidad de implementar, a nivel nacional, estrategias para disminuir el uso irracional de antibióticos, sobre todo en la población pediátrica. Es necesario complementar los datos de resistencia a penicilina con la determinación de la concentración mínima inhibitoria para hacer las recomendaciones terapéuticas respectivas.

Palabras clave: Streptococcus pneumoniae; Farmacorresistencia microbiana; Portador sano; Lactante (fuente DeCS BIREME).

\section{ANTIBIOTIC RESISTANCE OF Streptococcuspneumoniae AMONG HEALTHY NASOPHARYNGEAL CARRIERS IN SEVEN REGIONS OF PERU}

\begin{abstract}
Objectives. To determine the pattern of antibiotic susceptibility of isolated Streptococcus pneumoniae strains of healthy nasopharyngeal carriers younger than 2 years in seven regions of Peru. Materials and methods. Between 2007 and 2009 , nasopharyngeal swab samples were collected among 2123 healthy children aged 2-24 months in growth and development medical practices (CRED) and vaccination offices of hospitals and health centers in Lima, Piura, Cusco, Abancay, Arequipa, Huancayo, and lquitos. The resistance to ten antibiotics through disk diffusion sensitivity testing of isolated pneumococcus strains was determined. Results. 572 strains were isolated. High rates of resistance to co-trimoxazole (58\%), penicillin (52.2\% non-sensitive); tetracycline (29,1\%); azithromycin $(28,9 \%)$, and erythromycin (26,3\%). Resistance to chloramphenicol was low (8.8\%). Multiresistance was found at $29.5 \%$. Resistance to azithromycin and penicillin was different in all seven regions $(p<0,05)$, the highest percentage of non-sensitive strains being found in Arequipa $(63,6 \%)$, whereas the lowest percentage was found in Cusco (23.4\%). Conclusions. High levels of resistance found to penicillin, co-trimoxasole and macrolides in isolated pneumococcus strains of healthy carriers in all studied regions, and their association to a previous use of antibiotics, represent a significant public health problem in our country. This emphasizes the need to implement nationwide strategies to reduce the irrational use of antibiotics, especially among children. It is necessary to complement data of resistance to penicillin with the determination of minimal inhibitory concentration to make proper therapeutic recommendations.
\end{abstract}

Key words: Streptococcus pneumonia; Drug resistance, microbial; Carrier state; Infant (source: MeSH NLM).

\footnotetext{
Facultad de Medicina, Universidad Peruana Cayetano Heredia. Lima, Perú; ${ }^{2}$ Instituto de Medicina Tropical "Alexander von Humboldt", Universidad Peruana Cayetano Heredia. Lima, Perú; ${ }^{3}$ Instituto Nacional de Salud del Niño. Lima Perú; ${ }^{4}$ Hospital Nacional Cayetano Heredia. Lima, Perú; ${ }^{5}$ Hospital Nacional Edgardo Rebagliati Martins. Lima, Perú; ${ }^{6}$ Hospital Nacional Docente Madre Niño San Bartolomé. Lima, Perú; ${ }^{7}$ Hospital Nacional Daniel A. Carrión. Lima, Perú; ${ }^{8}$ Hospital Regional del Cusco. Cusco, Perú; ${ }^{9}$ Centro de Salud de Wanchaq. Cusco, Perú; ${ }^{10}$ Hospital Guillermo Díaz de la Vega. Abancay, Perú; ${ }^{11}$ Hospital EsSalud de Yanahuara. Arequipa, Perú; ${ }^{12}$ Policlínico Metropolitano de Arequipa. Arequipa, Perú; ${ }^{13}$ Department of Pediatrics and Child Health, University College Cork. Cork, Irlanda; ${ }^{14}$ Centro de Salud Urubamba. Cusco, Perú; ${ }^{15}$ Hospital de Emergencias Pediátricas. Lima, Perú; ${ }^{16}$ University of Texas School of Public Health. Texas, EE. UU.

a Médico cirujano; ${ }^{\mathrm{b}}$ biólogo; ${ }^{\mathrm{c}}$ tecnólogo médico; ${ }^{\mathrm{d}}$ médico infectólogo pediatra; ${ }^{\mathrm{e}}$ médico $\mathrm{PhD}$ en Microbiología. Recibido: 31-05-2013 Aprobado: 21-08-13
}

Citar como: Torres N, Velásquez R, Mercado EH, Egoavil M, Horna G, Mejía L, et al. Resistencia antibiótica de Streptococcus pneumoniae en portadores nasofaríngeos sanos de siete regiones del Perú. Rev Peru Med Exp Salud Publica. 2013;30(4):575-82. 


\section{INTRODUCCIÓN}

Streptococcus pneumoniae (neumococo) es un patógeno bacteriano responsable de una significativa morbilidad y mortalidad en niños y ancianos en todo el mundo. La Organización Mundial de la Salud (OMS) estima que alrededor de 700 mil niños menores de 5 años mueren cada año a nivel mundial a causa del neumococo, y que la mayor parte de ellos viven en países en vías de desarrollo (1). En Latinoamérica y el Caribe se estima que mueren anualmente entre 12 a 28 mil niños debido a enfermedad neumocócica; dicho de otra forma, de 2 a 3 niños mueren cada hora a causa de complicaciones ocasionadas por esta bacteria ${ }^{(2,3)}$.

S. pnemoniae es el responsable de graves enfermedades invasivas que pueden llegar a ser mortales como neumonía, sepsis y meningitis. En el año 2000, a nivel mundial se estimó un aproximado de 14,5 millones de episodios por año de enfermedad neumocócica invasiva en niños ${ }^{(4)}$. En el Perú, $S$. pneumoniae es uno de los patógenos más importantes asociados con neumonía y meningoencefalitis en niños ${ }^{(5)}$. Sin embargo, puede también ocasionar enfermedades no invasivas como otitis media aguda y sinusitis ${ }^{(6)}$. Se ha demostrado ampliamente que la colonización de la nasofaringe por $S$. pneumoniae es mayor durante los primeros dos años de vida ${ }^{(7)}$. El estado de portador es importante no solo porque es la condición inicial para desarrollar una enfermedad neumocócica invasiva, sino también porque proporciona la base para la expansión horizontal de este patógeno en la población ${ }^{(6,8)}$; y su estudio puede proporcionar información valiosa sobre las cepas circulantes en una comunidad.

La resistencia de $S$. pneumoniae a los antibióticos es un problema creciente. El desarrollo de cepas resistentes a penicilina se da gradualmente. La resistencia se puede deber a mutaciones genéticas repetidas de la bacteria y por presión selectiva por el uso indiscriminado de antibióticos ${ }^{(9,10)}$. En nuestro país los niveles de resistencia en portadores nasofaríngeos de $S$. pneumoniae a la penicilina han ido aumentando; sin embargo, los estudios se han limitado principalmente a Lima ${ }^{(11-14)}$.

En países donde se está introduciendo la vacuna conjugada contra el neumococo es necesario conocer la susceptibilidad antibiótica previa a la implementación de dicha vacuna, pues la resistencia antibiótica y distribución de los serotipos en los portadores nasofaríngeos se va a modificar con esta medida ${ }^{(15-17)}$. En un estudio previo, en el que se aislaron las cepas de $S$. pneumoniae, se describieron los serotipos circulantes en el país ${ }^{(18)}$. El objetivo del presente estudio fue determinar el patrón de susceptibilidad antibiótica de cepas de neumococo aisladas de portadores nasofaríngeos sanos menores de 2 años en siete regiones del Perú y determinar los factores asociados a la resistencia antibiótica.

\section{MATERIALES Y MÉTODOS}

La metodología utilizada para la selección de pacientes, muestreo e identificación de $S$. peumoniae están descritos en una publicación anterior de nuestro grupo ${ }^{(18)}$. En breve, este fue un estudio multicéntrico transversal realizado entre el 2007 y 2009, previo a la introducción de la vacuna conjugada contra el neumococo en nuestro país. Se tomaron muestras de hisopado nasofaríngeo en 2123 niños entre 2 meses y 2 años que acudieron a controles de crecimiento y desarrollo y vacunación de forma ambulatoria a establecimientos de salud de siete ciudades de Perú: Lima, Piura, Cusco, Abancay, Huancayo, Arequipa e lquitos.

Se tomaron muestras de hisopado nasofaríngeo a los niños, usando un hisopo delgado de alginato de calcio con vástago de aluminio (Fisher, Healthcare, Pittsburgh, $\mathrm{PA})$. Dichas muestras fueron tomadas por personal entrenado (pediatras o enfermeras). Se empleó como medio de transporte el medio Amies con carbón activado (Copan Innovation, Brescia, Italia), excepto en la ciudad de Piura, donde se empleó leche descremada triptona glucosa glicerol (LTGG), y en la ciudad de Lima, donde las muestras fueron sembradas directamente en placas de agar sangre, además de ser transportadas en caldo TSB (trypticase soy broth).

En el laboratorio central del proyecto, las muestras se sembraron en agar sangre (TSB suplementado con $5 \%$ de sangre de carnero). Se determinó el aislamiento como S. pneumoniae en base a la morfología de la colonia, alfa hemólisis, coloración Gram, solubilidad en bilis y sensibilidad a la optoquina. Las cepas identificadas como $S$. pneumoniae se guardaron a $-70{ }^{\circ} \mathrm{C}$ hasta su posterior envío a un laboratorio de referencia en un medio de transporte previamente validado (silica packs).

La determinación de serotipos de $S$. pneumoniae se realizó en el Laboratorio de Neumococo del Centro de Control y Prevención de Enfermedades (CDC) (Streptococcus pneumoniae Laboratory, Center for Disease Control and Prevention, Atlanta, EE. UU.). La serotipificación se realizó por el método de hinchamiento capsular (reacción de Quellung), empleando el esquema y los antisueros producidos en el CDC. 
Se determinó la sensibilidad a los siguientes antibióticos: penicilina (para lo cual se usó disco de oxacilina); eritromicina; azitromicina; cotrimoxazol; cloranfenicol; rifampicina; tetraciclina; clindamicina; vancomicina, y levofloxacino. Se utilizó la prueba de disco difusión (prueba de Kirby Bauer), que consiste en el sembrado de la cepa aislada de S. pneumoniae a una concentración de 0,5 en la escala de McFarland en un agar Mueller Hinton-sangre, sobre lo cual se coloca los discos de los antibióticos estudiados. Luego de $24 \mathrm{~h}$ se realiza la medición del diámetro de los halos correspondientes a cada antibiótico. Se utilizó los estándares de interpretación del Clinical and Laboratory Standards Institute (CLSI) para todos los antibióticos ${ }^{(19)}$. Todas las pruebas microbiológicas fueron realizadas en el Laboratorio de Microbiología del Instituto de Medicina Tropical "Alexander von Humboldt" de la Universidad Peruana Cayetano Heredia.

Se construyó una base de datos con la información clínica y de laboratorio en el programa Stata 10. Se realizó el análisis de resistencia antibiótica total, resistencia según grupo etario y resistencia según ciudad. Se utilizó la prueba del chi cuadrado con la corrección de Yates para el estudio de los factores asociados a la presencia de resistencia antibiótica y las comparaciones por ciudad.

Este estudio contó con la aprobación del Comité de Ética de la Universidad Peruana Cayetano Heredia y cada una de las instituciones participantes. Previa a la toma de muestras, se obtuvo el consentimiento informado de los padres.

\section{RESULTADOS}

Se analizaron 572 cepas de S. pneumoniae aisladas de la nasofaringe de niños sanos; 116 niños $(23,8 \%)$ presentaron alguna enfermedad respiratoria leve al momento de la toma de muestra; 78 (15,3\%) habían recibido algún antibiótico en los días previos, y 172 $(32,4 \%)$ habían recibido antibióticos dentro de los 3 meses previos (Tabla 1). La penicilina fue el antibiótico que con mayor frecuencia habían recibido los menores $(49,4 \%)$ (dentro de este grupo la amoxicilina fue la más usada, en un $96,5 \%$ ); seguido de cotrimoxazol $(12,2 \%)$; cefalosporinas $(9,3 \%)$, y macrólidos $(7,6 \%)$. En un $24,2 \%$ de los niños que habían consumido antibióticos, los familiares no recordaban el nombre del medicamento. Las ciudades con niños con menor uso previo de antibióticos fueron Huancayo y Cusco. El uso previo de antibióticos al momento de la toma de muestra y 3 meses antes, así como el antecedente de hospitalización previa, fueron significativamente diferentes en las ciudades estudiadas (Tabla 1).

El antibiótico al que fue resistente el mayor porcentaje de cepas fue cotrimoxazol (58\%), seguido por penicilina (52,2\% no-sensibles), tetraciclina $(29,1 \%)$, y azitromicina

Tabla 1. Características de los niños portadores de Streptococcus pneumoniae por región

\begin{tabular}{|c|c|c|c|c|c|c|c|c|}
\hline & $\begin{array}{c}\text { Lima } \\
\mathrm{n}=314\end{array}$ & $\begin{array}{l}\text { Iquitos } \\
n=59\end{array}$ & $\begin{array}{l}\text { Piura } \\
n=48\end{array}$ & $\begin{array}{c}\text { Cusco } \\
n=47\end{array}$ & $\begin{array}{c}\text { Abancay } \\
n=42\end{array}$ & $\begin{array}{c}\text { Arequipa } \\
n=33\end{array}$ & $\begin{array}{c}\text { Huancayo } \\
n=29\end{array}$ & $\begin{array}{c}\text { Total } \\
n=572\end{array}$ \\
\hline $\begin{array}{l}\text { Edad } \\
\text { mediana (p25, p75) }\end{array}$ & $\begin{array}{c}12 \\
(7,17)\end{array}$ & $\begin{array}{c}8 \\
(5,11)\end{array}$ & $\begin{array}{c}9 \\
(6,18)\end{array}$ & $\begin{array}{c}12 \\
(6,16)\end{array}$ & $\begin{array}{c}14,5 \\
(7,18)\end{array}$ & $\begin{array}{c}15 \\
(11,18)\end{array}$ & $\begin{array}{c}10 \\
(6,13)\end{array}$ & $\begin{array}{c}12 \\
(7,17)\end{array}$ \\
\hline $\begin{array}{l}\text { Número de personas en el hogar } \\
\text { mediana (p25, p75) }\end{array}$ & $\begin{array}{c}5 \\
(4,7)\end{array}$ & $\begin{array}{c}5 \\
(4,7)\end{array}$ & $\begin{array}{c}5 \\
(4,8)\end{array}$ & $\begin{array}{c}5 \\
(3,6)\end{array}$ & $\begin{array}{c}5 \\
(4,7)\end{array}$ & $\begin{array}{c}5 \\
(4,6)\end{array}$ & $\begin{array}{c}4 \\
(4,5)\end{array}$ & $\begin{array}{c}5 \\
(4,7)\end{array}$ \\
\hline Femenino & 43,6 & 47,5 & 54,2 & 61,7 & 47,6 & 48,5 & 42,8 & 47,0 \\
\hline Masculino & 56,4 & 52,5 & 45,8 & 38,3 & 52,4 & 51,5 & 57,2 & 53,0 \\
\hline \multicolumn{9}{|l|}{ Enfermedad respiratoria leve al momento (\%) } \\
\hline Presente & 26,0 & 23,8 & 25,0 & 29,8 & 19,5 & 12,1 & 10,3 & 23,8 \\
\hline Ausente & 74,0 & 76,2 & 75,0 & 70,2 & 80,5 & 87,9 & 89,7 & 76,2 \\
\hline \multicolumn{9}{|l|}{ Uso previo de antibióticos (\%) } \\
\hline Presente & 18,5 & 15,3 & 6,3 & 2,1 & 26,2 & 15,6 & 0.0 & $15,2^{*}$ \\
\hline \multicolumn{9}{|c|}{ Uso de antibióticos en los tres últimos meses (\%) } \\
\hline Presente & 36,2 & 36,4 & 41,7 & 14,9 & 19,0 & 36,4 & 17,2 & $32,4^{\dagger}$ \\
\hline Ausente & 63,8 & 63,6 & 58,3 & 85,1 & 81,0 & 63,6 & 82,8 & 67,6 \\
\hline \multicolumn{9}{|l|}{ Asistencia a guardería (\%) } \\
\hline Asiste & 3,9 & 0,0 & 6,3 & 2,2 & 2,4 & 3,3 & 0,0 & 3,4 \\
\hline No asiste & 96,1 & 100,0 & 93,7 & 97,8 & 97,6 & 96,7 & 100,0 & 96,6 \\
\hline \multicolumn{9}{|l|}{ Hospitalización previa (\%) } \\
\hline Presente & 27,5 & 18,2 & 6,3 & 8,5 & 7,1 & 25,8 & 3,5 & $20,4^{\dagger}$ \\
\hline Ausente & 72,5 & 81,8 & 93,7 & 91,5 & 92,9 & 74,2 & 96,5 & 79,6 \\
\hline
\end{tabular}

${ }^{*} p<0,05 ;{ }^{\dagger} p<0,01$ para la comparación entre las diferentes ciudades 
Tabla 2. Sensibilidad antibiótica de Streptococcus pneumoniae en niños portadores sanos de siete regiones del Perú.

\begin{tabular}{|c|c|c|c|}
\hline Antibiótico & $\begin{array}{c}\text { Sensible } \\
\text { n (\%) }\end{array}$ & $\begin{array}{c}\text { Intermedio } \\
\text { n (\%) }\end{array}$ & $\begin{array}{c}\text { Resistente } \\
\text { n (\%) }\end{array}$ \\
\hline Cotrimoxazol $(n=566)$ & $196(34,6)$ & $42(7,4)$ & $328(58,0)$ \\
\hline Penicilina $(n=565)$ & $270(47,8)$ & - & $295(52,2)^{*}$ \\
\hline Tetraciclina $(n=570)$ & $396(69,5)$ & $8(1,4)$ & $166(29,1)$ \\
\hline Azitromicina $(n=519)$ & $360(69,4)$ & $9(1,7)$ & $150(28,9)$ \\
\hline Eritromicina $(n=570)$ & $407(71,4)$ & $13(2,3)$ & $150(26,3)$ \\
\hline Clindamicina $(n=570)$ & $489(85,8)$ & $9(1,6)$ & $72(12,6)$ \\
\hline Cloranfenicol $(n=567)$ & $517(91,2)$ & - & $50(8,8)$ \\
\hline Rifampicina $(n=569)$ & $564(99,1)$ & $0(0)$ & $5(0,9)$ \\
\hline Levofloxacino $(n=568)$ & $568(100)$ & $0(0)$ & $0(0)$ \\
\hline Vancomicina $(n=570)$ & $570(100)$ & $0(0)$ & $0(0)$ \\
\hline
\end{tabular}

*No-sensible a penicilina

$(28,9 \%)$. La resistencia a cloranfenicol fue baja $(8,8 \%)$ (Tabla 2). El 28,9\% de las cepas fueron pansensibles; el $15,4 \%$ fueron resistentes a un solo antibiótico y el $29,5 \%$ fueron multirresistentes (resistentes a tres o más grupo de antibióticos). Se encontraron 19 patrones de multirresistencia. El patrón de resistencia más frecuente fue a cinco tipos de antibióticos: cotrimoxazol, penicilina, tetraciclina, eritromicina/azitromicina y clindamicina, presente en 44 cepas $(7,7 \%)$, seguido de resistencia solo a cotrimoxasol, penicilina y eritromicina/azitromicina, presente en 28 cepas (4,9\%). La resistencia a estos tres antibióticos simultáneamente, asociada o no a resistencia a otros antibióticos, estuvo presente en 112 cepas $(19,6 \%)$.

La resistencia antibiótica por regiones tuvo una distribución similar. En todas las regiones los antibióticos a los que más cepas presentaron resistencia fueron cotrimoxazol y penicilina. La mayor resistencia a cotrimoxazol se observó en Piura $(77,1 \%)$ seguido de Huancayo $(65,5 \%)$, mientras que el mayor porcentaje de cepas no-sensibles a penicilina se encontró en Arequipa $(63,6 \%)$ y Lima $(57,4 \%)$. En general, la región con menor resistencia a todos los antibióticos evaluados fue Cusco, presentando solo $36,2 \%$ de resistencia a cotrimoxazol, 23,4\% (no-sensible) a penicilina y $10,6 \%$ a azitromicina, siendo estos porcentajes significativamente diferentes con la resistencia en el resto de ciudades $(p<0,01)$ (Figura 1). La no-sensibilidad a penicilina fue significativamente diferente en las siete regiones $(p<0,05)$, así como la resistencia a azitromicina $(p<0,01)$; mientras que la resistencia a cotrimoxazol no fue significativamente diferente $(p=0,48)$

Con respecto a la resistencia según grupos etarios, esta fue mayor en los niños mayores de 1 año comparado con los menores de 1 año, siendo esta diferencia significativa para cotrimoxazol $(p=0,01)$, penicilina $(p=0,001)$, azitromicina $(p=0,01)$ y eritromicina $(p=0,02)$ (Figura 2).
El análisis de los factores asociados a cepas no-sensibles a penicilina mostró asociación significativa con uso previo de antibióticos, hospitalización anterior y presencia de más de siete habitantes en el hogar (Tabla 3). El 58,4\% de niños que usaron antibióticos previamente portaban cepas de neumococo no-sensibles a penicilina, en comparación con el $48,4 \%$ de niños que no habían usado antibióticos previamente $(p=0,03)$. El $72 \%$ de niños que asistieron a guarderías portaron cepas no-sensibles a penicilina versus el $50,5 \%$ de los que no asistían, no siendo esta diferencia significativa, dado el reducido número de niños que asistían a guarderías en general (3,4\% del total de niños enrolados). Sin embargo, la asistencia a guarderías sí estuvo asociada a portar cepas resistentes a azitromicina/eritromicina $(p=0,01)$ y a tetraciclina $(p=0,01)$. No se encontraron factores asociados a portar cepas resistentes a cotrimoxazol.

En base a la serotipificación de 526 cepas, publicada previamente ${ }^{(18)}$, los serotipos más frecuentes fueron: $19 \mathrm{~F}(18,1 \%) ; 6 \mathrm{~B}(14,3 \%) ; 23 \mathrm{~F}(8,9 \%)$, y 14 (6,5\%). Entre las

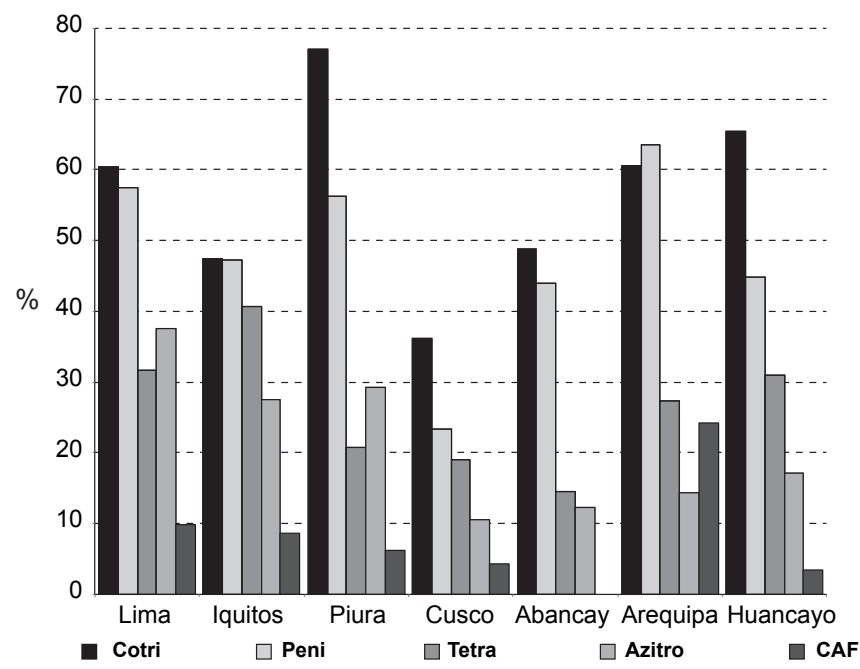

Figura 1. Resistencia antibiótica de Streptococcus pneumoniae según región del Perú, por disco Kirby-Bauer Cotri: cotrimoxazol, Peni: penicilina, Tetra: tetraciclina, Azitro: azitromicina, CAF: cloranfenicol 


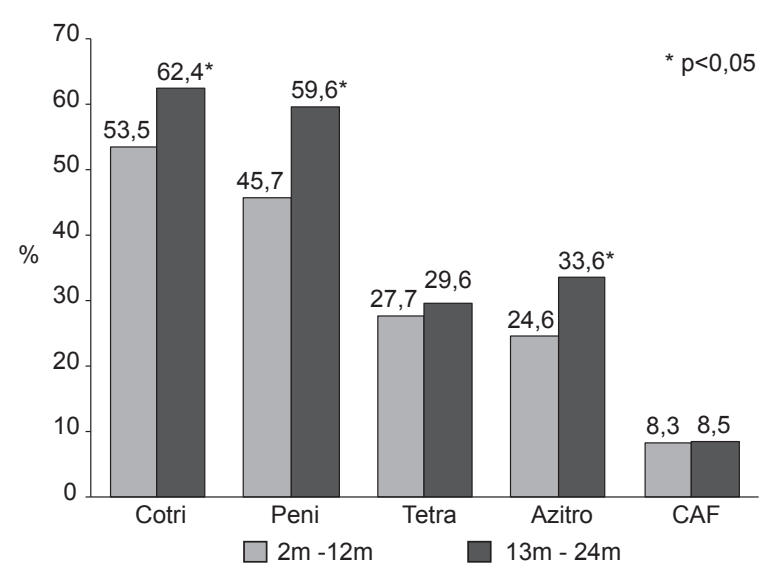

Figura 2. Resistencia antibiótica de Streptococcus pneumoniae por grupo etario.

Cotri: cotrimoxazol, Peni: penicilina, Tetra: tetraciclina, Azitro: azitromicina, CAF: Cloranfenicol

cepas no sensibles a penicilina, las más frecuentes fueron: $19 F(29 \%) ; 6 B(20,2 \%)$, y $14(11,9 \%)$ (Figura 3$)$. Por otro lado, los serotipos con mayor porcentaje de no-sensibilidad a penicilina fueron: $14(88,2 \%)$; $19 \mathrm{~F}(76,9 \%)$, y $6 \mathrm{~B}(68,9)$. El serotipo $19 \mathrm{~A}$ presentó $60 \%$ de no-sensibilidad a penicilina y $40 \%$ de resistencia a cotrimoxazol. Adicionalmente, se encontró un 30,5\% de cepas no-sensibles a penicilina en los serotipos no presentes en la vacunas heptavalente $(4,6 \mathrm{~B}, 9 \mathrm{~V}, 14,18 \mathrm{C}, 19 \mathrm{~F}, 23 \mathrm{~F})$ y decavalente $(1,4,5,6 \mathrm{~B}$, 7F, 9V, 14, 18C, 19F, 23F).

\section{DISCUSIÓN}

Este estudio muestra la presencia de un elevado porcentaje de cepas de $S$. pneumoniae resistentes a

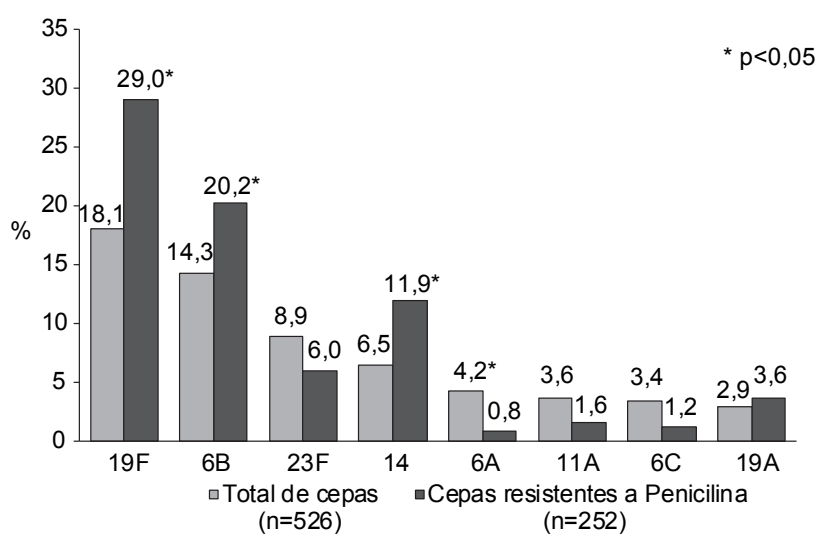

Figura 3. Distribución de los principales serotipos en el total de cepas versus las cepas no-sensibles a penicilina

los principales antibióticos usados en pediatría, no solo en Lima, sino también en otras ciudades del interior del país. La tasa de resistencia al cotrimoxazol fue la más alta, con valores similares a lo reportado en un estudio previo en Lima, y semejante a lo descrito en otros países de Latinoamérica y del mundo, donde se reporta una resistencia entre 40 y $77 \%{ }^{(20-22)}$. Esto convierte al cotrimoxazol en una mala elección para combatir las infecciones causadas por el neumococo, pese a ser el antibiótico más ampliamente usado para infecciones respiratorias en centros del primer nivel de atención en nuestro país.

La resistencia a la penicilina ha ido aumentando con el tiempo. Estudios previos en niños portadores sanos de Lima reportaron $5 \%$ de cepas resistentes a la penicilina

Tabla 3. Factores asociados a la falta de suceptibilidad antibiótica de Streptococcus pneumoniae a penicilina y a macrólidos

\begin{tabular}{|c|c|c|c|c|c|c|c|c|}
\hline & \multicolumn{4}{|c|}{ Penicilina } & \multicolumn{4}{|c|}{ Eritromicina / Azitromicina } \\
\hline & S (\%) & $\mathbf{R}^{*}(\%)$ & OR (IC 95\%) & p & $\mathbf{S}$ & $\mathbf{R}$ & OR (IC 95\%) & p \\
\hline \multicolumn{9}{|c|}{ Uso de antibióticos previo } \\
\hline No & 51,6 & 48,4 & $1,50(1,03-2,16)$ & 0,03 & 73,2 & 26,8 & $1,11(0,77-1,72)$ & 0,49 \\
\hline $\mathrm{Si}$ & 41,6 & 58,4 & & & 70,3 & 29,7 & & \\
\hline \multicolumn{9}{|c|}{ Asistencia a guardería } \\
\hline No & 49,5 & 50,5 & $2,55(0,89-7,26)$ & 0,07 & 70,9 & 29,1 & $0,10(0,05-0,20)$ & 0,01 \\
\hline $\mathrm{Si}$ & 27,8 & 72,2 & & & 41,2 & 58,8 & & \\
\hline \multicolumn{9}{|c|}{ Hospitalización previa } \\
\hline No & 51,0 & 49,0 & $1,58(1,03-2,44)$ & 0,04 & 73,1 & 26,9 & $0,48(0,31-0,75)$ & 0,54 \\
\hline $\mathrm{Si}$ & 39,6 & 60,4 & & & 70,1 & 29,9 & & \\
\hline \multicolumn{9}{|c|}{ Niños $<6$ años en el hogar } \\
\hline Menos de 4 & 48,1 & 51,9 & $1,39(0,56-3,46)$ & 0,48 & 70,9 & 29,1 & $0,02(0,01-0,02)$ & 0,05 \\
\hline 4 a más & 40,0 & 60,0 & & & 90,5 & 9,5 & & \\
\hline \multicolumn{9}{|c|}{ Número personas en el hogar } \\
\hline Menos de 7 & 51,1 & 48,9 & $1,51(1,03-2,21)$ & 0,04 & 69,4 & 30,6 & $0,71(0,46-1,08)$ & 0,76 \\
\hline 7 a más & 40,9 & 59,1 & & & 70,8 & 29,2 & & \\
\hline
\end{tabular}

$\mathrm{S}$, sensible; R, resistente; *no-sensible a penicilina 
en $1997^{(20)} ; 15 \%$ en el 2000 y $2001^{(12,13) ;} 36$ y $37 \%$ en el 2003 y 2004 , respectivamente ${ }^{(12,23)}$; y $47 \%$ en el $2008^{(24)}$. En estudios en pacientes con enfermedad neumococcica invasiva en el Perú se reportó 3\% de resistencia a la penicilina en adultos en $1994{ }^{(25)}$; $27 \%$ en niños en el $2000{ }^{(14) ;}$ y $23 \%$ en el $2008{ }^{(5)}$. Este incremento también se ha evidenciado en otros países de Latinoamérica (Brasil, Argentina, Chile y Colombia) ${ }^{(21,26)}$ en estudios realizados antes de la introducción de la vacuna conjugada contra el neumococo en los programas nacionales de inmunizaciones. Sin embargo, es necesario complementar estos datos con la determinación de la concentración mínima inhibitoria (CMI) para poder hacer recomendaciones terapéuticas, dado que para los datos del presente trabajo solo se usó la prueba del disco, lo que no permite discriminar entre resistencia alta o intermedia. Por otro lado, las guías internacionales establecen los estándares de interpretación de cepas meníngeas y no meníngeas de $S$. pneumoniae basados en la CMI. En el caso de la penicilina, para cepas meníngeas (aisladas de pacientes con meningitis), se considera sensible si la $\mathrm{CMI}$ es $\leq 0,06 \mu \mathrm{g} / \mathrm{mL}$ y resistente si el $\mathrm{CMI}$ es $\geq 0,12 \mu \mathrm{g} / \mathrm{mL}$; mientras que para cepas no-meníngeas (por ejemplo para casos de neumonía) se considera sensible si la CMI es $\leq 2 \mu \mathrm{g} / \mathrm{mL}$ y resistente si la $\mathrm{CMI}$ es $\geq 8 \mu \mathrm{g} / \mathrm{mL}{ }^{(19)}$. Sin embargo, los datos de resistencia por disco, correlacionan muy bien con los niveles de resistencia para cepas meníngeas, mas no así para cepas no meníngeas (neumonía, bacteremia). Por lo tanto, en base a estos estudios, podríamos recomendar a nivel nacional no usar penicilina para los casos de meningitis en los que se sospecha neumococo; en estos casos de debe usar de manera empírica ceftriaxona u otra cefalosporina de tercera generación o cloranfenicol. Por el contrario, en el caso de neumonía en el que se sospecha neumococo, las penicilinas (incluyendo la amoxicilina) siguen siendo la droga de elección para el tratamiento, dado que la resistencia en estos casos es muy baja. Esta recomendación se basa en el estudio previo de nuestro grupo en cepas invasivas de neumococo en Lima, en el que encontramos $46,2 \%$ de resistencia a penicilina en cepas meníngeas y solo $8,1 \%$ de resistencia en cepas no-meníngeas (incluyendo $6,5 \%$ de resistencia alta y $1,6 \%$ de resistencia intermedia), mediante la determinación de la $\mathrm{CMI}{ }^{(5)}$.

Con relación a la edad, se encontró un mayor porcentaje de cepas resistentes en niños de mayor edad, lo que podría deberse a un mayor uso de antibióticos y mayor exposición a otros portadores, todo lo que aumenta la posibilidad de colonización por cepas resistentes, tal como ha sido descrito anteriormente ${ }^{(8-10)}$. En nuestro estudio las cepas aisladas de niños de la costa (Lima, Piura) mostraron mayor resistencia antibiótica en comparación con las cepas asiladas de niños de la sierra (Cusco y Abancay). La resistencia antibiótica de cepas asiladas en el Cusco fue significativamente menor en comparación con las otras regiones para todos los antibióticos evaluados. Esto podría deberse a un menor uso de antibióticos en esta región; sin embargo, no se pudo realizar el análisis de factores asociados a resistencia para cada una de las regiones, dado el reducido número de cepas aisladas en cada ciudad. Al analizar los factores asociados a no-sensibilidad a penicilina y resistencia a macrólidos, para el total de cepas, se encontró asociación con uso previo de antibióticos, asistencia a guarderías y hacinamiento, factores que han sido ampliamente descritos en la literatura $(9,10,22,27)$.

Otra de las limitaciones del estudio, además de que no se pudo determinar la CMI, fue la ausencia de datos de algunos niños, principalmente para el análisis de los factores asociados a resistencia antibiótica y el reducido número de cepas en cada región para poder estudiar los factores asociados a resistencia en cada zona.

En conclusión, los elevados niveles de resistencia encontrados para penicilina, cotrimoxazol y macrólidos en todas las regiones estudiadas, y su asociación con uso previo de antibióticos, representan un importante problema de salud pública en nuestro país. Esto resalta la necesidad de implementar a nivel nacional estrategias para disminuir el uso irracional de antibióticos, sobre todo en la población pediátrica. Como ya está descrito en la literatura ${ }^{(27)}$, un control adecuado del uso de antibióticos puede contribuir a una disminución significativa de los patrones de resistencia antibiótica en una población. Las estrategias de control de uso de antibióticos incluyen, en primer lugar, una adecuada y permanente capacitación a los médicos y demás personal de salud, resaltando de manera especial el rol predominante de las infecciones virales en las infecciones respiratorias altas, neumonía y diarrea en niños pequeños, así como en la adecuada selección de los antibióticos y la duración de su prescripción, cuando están indicados. En segundo lugar se requiere de regulaciones para la venta de antibióticos en las farmacias; y en tercer lugar, educación de la población para evitar la automedicación. Con estas medidas empezaremos a disminuir la emergencia y diseminación de cepas resistentes en nuestro país. Adicionalmente, dado que este estudio fue realizado antes de la introducción de las vacunas conjugadas contra S. pneumoniae en el Perú, se requiere de estudios posteriores para evaluar el impacto de la vacunación en la dinámica de las cepas resistentes.

Agradecimientos: a Benita Larota y Dora Leandro (Instituto de Medicina tropical Alexander von Humboldt, Universidad 
Peruana Cayetano Heredia, Lima); Jaime Solís Esperanza Ticona y Rosminy Ayquipa (Hospital Guillermo Diaz de la Vega, Abanzay); Felicitas Hanco, Diomar Aedo (Hospital Essalud Cusco, Centro de Salud Wanchaq, Cusco); Luis Porras, Andres Barriga, Fredy Camayo y Jenny Arteaga (Hospital El Carmen y Hospital Daniel Alcides Carrión, Huancayo); Miguel Mestanza y Jorge Diaz (Hospital Regional y Hospital Essalud, Iquitos); Jorge Frias, Giovanna Wong, Oscar Requena y Victor Velarde (CLAS Catacaos, Hospital de Essalud de Sullana, Hospital Santa Rosa y Hospital Regional Cayetano Heredia, Piura), por su colaboración en la ejecución del estudio.

Contribuciones de autoría: NT, RV, EM, ME, MEC, EC, RH, WS, IR y TJO participaron en la concepción y diseño del artículo. ME, GH, LM, MEC, EC, RH, WS, FEC, AS, IR, FH, CL, AV, RC, $\mathrm{BL}, \mathrm{BT}, \mathrm{FC}, \mathrm{AC}, \mathrm{SR}$, EL aportaron con pacientes para el estudio. NT, RV, EM ME, GH, MEC, EC, RH, WS, FEC, AS, IR, FH, CL, $A V, R C, B L$ y TJO participaron en la recolección/obtención de resultados. NT, RV, EM, y TJO analizaron e interpretaron datos. NT, RV, EM, JP y TJO redactaron el artículo. NT, RV, MEC y TJO obtuvieron financiamiento. LM y HG asesoraron técnicamente en el estudio. Todos los autores revisaron en forma crítica las versiones del manuscrito y aprobaron su versión final.

Fuentes de financiamiento: Fondo de Apoyo a la Investigación de la Facultad de Medicina Alberto Hurtado, Universidad Peruana Cayetano Heredia y por el Fondo de Apoyo a la Investigación del Instituto Nacional de Salud de Niño.

Conflictos de interés: TJO, EC, GH, WS, RH, MEC, ME, AS y FEC han recibido financiamiento por el Laboratorio Wyeth (actualmente Pfizer) para el estudio de cepas invasivas de $S$. pneumoniae. EHM recibió entrenamiento en serotipificación de neumococo en el CDC a través del proyecto "Global Strain Bank Project" financiado por PATH (Partnership Action on Tobacco and Health).

\section{REFERENCIAS BIBLIOGRÁFICAS}

1. Pneumococcal conjugate vaccine for childhood immunization - WHO position paper. Wkly Epidemiol Rec. 2007;82(12):93-104.

2. Valenzuela ME, Flannery B. La epidemiología numocócica global. En: Organización Panamericana de la Salud. Acta del Segundo Simposio Regional sobre Neumococo. Procedente del II Simposio Regional de Enfermedad Neumocócica. 13 y 14 de diciembre de 2006. Sao Paulo, Brasil. Geneva: OPS;2006.

3. Valenzuela MT, O’Loughlin R, De La Hoz F, Gomez F, Constenla D, Sinha A, et al. The burden of pneumococcal disease among Latin American and Caribbean children: review of the evidence. Rev Panam Salud Publica. 2009;25(3):270-9.

4. O'Brien K, Wolfson LJ, Watt JP, Henkle E, Deloria-Knoll M, McCall $\mathrm{N}$, et al. Burden of disease caused by Streptococcus pneumoniae in children younger than 5 years: global estimates. Lancet. 2009;374(9693):893-902. doi: 10.1016/S0140-6736(09)61204-6.

5. Ochoa TJ, Egoavil M, Castillo ME, Reyes I, Chaparro E, Silva W, et al. Invasive pneumococcal diseases among hospitalized children in Lima, Peru. Rev Panam Salud Publica. 2010;28(2):121-7.

6. Faden H, Duffy L, Wasielewski R, Wolf J, Krystofik D, Tung Y. Relationship between nasopharyngeal colonization and the development of otitis media in children. J Infect Dis. 1997;175(6):1440-5.

7. Lloyd-Evans N, O'Dempsey TJ Baldeh I, Secka O, Demba E, Todd JE, et al. Nasopharyngeal carriage of pneumococci in Gambian children and in their families. Pediatr Infect Dis J. 1996;15(10):866-71.

8. Bogaert D, De Groot R, Hermans PW. Streptococcus pneumoniae colonisation: the key to pneumococcal disease. Lancet Infect Dis. 2004;4(3):144-54.

9. Melander E, Môlstad S, Persson K, Hansson HB, Sôderstrôm M, Ekdahl K. Previous antibiotic consumption and other risk factors for carriage of penicillin-resistant Streptococcus pneumoniae in children. Eur J Clin Microbiol Infect Dis. $1998 ; 17(12): 834-8$.

10. Melander E, Ekdahl K, Jônsson G, Môlstad S. Frequency of penicillinresistant pneumococci in children is correlated to community utilization of antibiotics. Pediatr Infect Dis J. 2000;19(12):1172-7.

11. Diaz S, Morales S, Gonzalez D, Salazar E. Serotipos de Streptococcus pneumoniae aislados de procesos invasivos, 1999-Abril 2000. En: VII Congreso Peruano de Enfermedades Infecciosas y Tropicales; 2001 Aug 30-Sep 2; Lima, Peru. Universidad Nacional Mayor de San Marcos, 2001; Abstract $N^{\circ}$ ON11.

12. Ochoa TJ, Rupa R, Guerra $H$, Hernandez H, Chaparro E, Tamariz $\mathrm{J}$, et al. Penicillin resistance and serotypes/serogrups of Streptococcus pneumoniae in nasopharyngeal carrier children younger than 2 years in Lima, Peru. Diagnostic Microbiol Infect Dis. 2005;52(1):59-64.

13. Cullota AR, Kalter HD, Delgado J, Gilman RH, Facklam RR, Velapatino B, et al. Antimicrobial susceptibilities and serotype distribution of Streptococcus pneumoniae isolates from a low socioeconomic area in Lima, Peru. Clin Diagn Lab Immunol. 2002;9(6):1328-31.

14. Morales S, Díaz S, González D, Huapaya B. Susceptibilidad antimicrobiana de Streptococcus pneumoniae determinando la concentración inhibitoria mínima, 1999. Rev Med ExpSaludPublica. 2001;18(1-2)35-37.

15. Tóthpál A, Ordas A, Hajdú E, Kardos S, Nagy E, Nagy K, et al. A marked shift in the serotypes of pneumococci isolated from healthy children in Szeged, Hungary, over a 6-year period. Acta Microbiol Immunol Hung. 2011;58(3):239-46. doi: 10.1556/ AMicr.58.2011.3.8.

16. Dagan R, Givon-Lavi N, Zamir O, Fraser D. Effect of nonavalent conjugate vaccine on carriage of antibioticresistance Streptococcus pneumoniae in day-care centers. Pediatr Infect Dis J. 2003;22(6):532-40.

17. Isaacman DJ, McIntosh ED, Reinert RR. Burden of invasive pneumococcal disease and serotype distribution among Streptococcus pneumoniae isolates in young children in Europe: 
impact of 7-valent pneumococcal conjugate vaccine and considerations for future conjugate vaccines. Int J Infect Dis. 2010;14(3):e197-209. doi: 10.1016/j.ijid.2009.05.010.

18. Mercado EH, Ochoa TJ, Egoavil M, Horna G, Torres N, Velasquez R, et al. Serotipos de neumococo en portadores nasofaríngeos en niños peruanos previo a la introducción de las vacunas conjugadas. Rev Peru Med Exp Salud Publica. 2012;29(1):53-60.

19. Clinical and Laboratory and Standars Institute (CLSI). Performance standards for antimicrobial susceptibility testing. Eighteenth informational supplement. M100-S18. Wayne: CLSI; 2008.

20. Ochoa Woodell TJ. Resistencia de Streptococcus pneumoniae a penicilina en portadores nasofaríngeos menores de 2 años. Rev Med Hered. 1998;9(2):56-62.

21. Castanheira M, Gales AC, Mendes RE, Jones RN, Sader HS. Antimicrobial susceptibility of Streptococcus pneumoniae in Latin America: results from five years of the SENTRY Antimicrobial Surveillance Program. Clin Microbiol Infect. 2004;10(7):645-51.
22. Saha SK, Baqui AH, Darmstadt GL, Ruhulamin M, Hanif M, El Arifeen $S$, et al. Comparison of antibiotic resistance and serotype composition of carriage and invasive pneumococci among Bangladeshi children: implications for treatment policy and vaccine formulation. J Clin Microbiol. 2003;41(12):5582-7.

23. Guevara J, Ocsas J, Valencia E, Rojas J, Flores P. Prevalencia y resistencia de Neumococos en una población escolar aparentemente sana. Rev Per Med Trop. 2004;19(1):59-63.

24. Velásquez ER, Torres N, Horna G, Pando J, Castillo ME, Hernández $\mathrm{R}$, et al. Sensibilidad antibiótica de Streptococcus pneumoniae en portadores nasofaríngeos en niños menores de un año en Lima, Perú. Acta Med Peruana. 2008;25(3):148-52.

25. Fukuda J, Echevarría J, Llanos F, Yi A, Palomino S, Gotuzzo E, et al. Streptococcus pneumoniae resistentes a la penicilina en Lima - Perú. Rev Med Hered. 1996;7(1):11-16.

26. Di Fabio JL, Castañeda E, Agudelo CI, De La Hoz F, Hortal M, Camou T, et al. Evolution of Streptococcus pneumoniae serotypes and penicillin susceptibility in Latin America, Sireva-Vigía Group, 1993 to 1999. Pediatr Infect Dis J. 2001;20(10):959-67.

27. Van Eldere J, Mera RM, Miller LA, Poupard JA, Amrine-Madsen H. Risk factors for development of multipleclass resistance to Streptococcus pneumoniae Strains in Belgium over a 10-year period: antimicrobial consumption, population density, and geographic location. Antimicrob Agents Chemother. 2007;51(10):3491-7.

Correspondencia: Theresa J. Ochoa Dirección: Instituto de Medicina Tropical "Alexander von Humboldt", Universidad Peruana Cayetano Heredia, Av. Honorio Delgado, Lima 31, Perú. Teléfono: 51-1-482-3910

Correo electrónico: Theresa.J.Ochoa@uth. tmc.edu

\section{Consulte las ediciones anteriores de la Revista Peruana de Medicina Experimental y Salud Pública en www.scielosp.org}

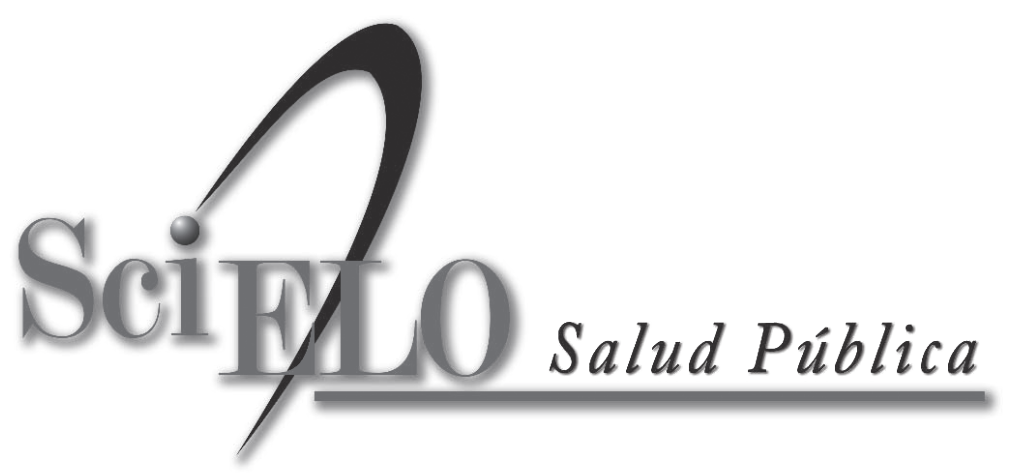

